\title{
Diagnosis and Management of Tuberculous Pericarditis: What Is New?
}

\author{
Godsent Isiguzo ${ }^{1,2} \cdot$ Elsa Du Bruyn ${ }^{3} \cdot$ Patrick Howlett $^{3,4} \cdot$ Mpiko Ntsekhe $^{5}$ \\ Published online: 15 January 2020 \\ (C) Springer Science+Business Media, LLC, part of Springer Nature 2020
}

\begin{abstract}
Purpose of Review This review provides an update on the immunopathogenesis of tuberculous pericarditis (TBP), investigations to confirm tuberculous etiology, the limitations of anti-tuberculous therapy (ATT), and recent efficacy trials.

Recent Findings A profibrotic immune response characterizes TBP, with low levels of AcSDKP, high levels of $\gamma$-interferon and IL-10 in the pericardium, and high levels of TGF- $\beta$ and IL-10 in the blood. These findings may have implications for future therapeutic targets. Despite advances in nucleic acid amplification approaches, these tests remain disappointing for TBP. Trials of corticosteroids and colchicine have had mixed results, with no impact on mortality, evidence of a reduction in rates of constrictive pericarditis and potential harm in those with advanced HIV. Small studies suggest that ATT penetrates the pericardium poorly. Given that there is a close association between high bacillary burden and mortality, a rethink about the optimal drug doses and duration may be required.

Summary The high mortality and morbidity from TBP despite use of anti-tuberculous drugs call for researches targeting hostdirected immunological determinants of treatment outcome. There is also a need for the identification of steps in clinical management where interventions are needed to improve outcomes.
\end{abstract}

Keywords Tuberculous pericarditis · Mycobacterium tuberculosis

\section{Case Vignette}

A 33-year-old woman, HIV positive with a CD4 count of 21 cells $/ \mu \mathrm{L}$, presented with weight loss, night sweats, and shortness of breath. At presentation, recorded examination

This article is part of the Topical Collection on Pericardial Disease

Electronic supplementary material The online version of this article (https://doi.org/10.1007/s11886-020-1254-1) contains supplementary material, which is available to authorized users.

Godsent Isiguzo

isggod002@myuct.ac.za

Elsa Du Bruyn

elsa.dubruyn@uct.ac.za

Patrick Howlett

patrick.howlett@gmail.com

Mpiko Ntsekhe

mpiko.ntsekhe@uct.ac.za

1 Department of Medicine, University of Cape Town,

Cape Town, South Africa findings were temperature of $35.5^{\circ} \mathrm{C}$, peripheral oxygen saturation $100 \%$, respiratory rate $22 / \mathrm{min}$, heart rate 112 beats/ min, blood pressure 106/62 $\mathrm{mmHg}$ with a pulsus paradoxus of $16 \mathrm{mmHg}$, and distant heart sounds. Her admission electrocardiogram (ECG), chest radiograph (CXR), and point of
2 Department of Medicine, Federal Teaching Hospital Abakaliki, Abakaliki, Nigeria

3 Wellcome Centre for Infectious Diseases Research in Africa, Institute of Infectious Disease and Molecular Medicine, and Department of Medicine, University of Cape Town, Cape Town 7925, Republic of South Africa

4 Department of Medicine, Imperial College, Kensington, London SW7 2DD, UK

5 Division of Cardiology, Groote Schuur Hospital and Faculty of Health Sciences, University of Cape Town South Africa, Cape Town, South Africa 
care echocardiogram (parasternal long axis, four chamber, and subcostal views) are presented.

Her echocardiogram showed a swinging heart surrounded by a large pericardial effusion with strands and right ventricular collapse (see Supplementary Figures 1-4). Doppler studies across the mitral valve inflow revealed significant inspiratory E wave variation. There was IVC plethora with less than $50 \%$ collapse in inspiration, and the diagnosis of TBP with cardiac tamponade was made.

She proceeded to undergo pericardiocentesis with $1100 \mathrm{~mL}$ of bloodstained fluid removed from her pericardium. She was commenced on anti-TB treatment (ATT), and 2 weeks later, on anti-retroviral therapy (ART). At the 6-month follow-up visit, the patient had completed ATT, CD4 count was 252 cells $/ \mu \mathrm{L}$, and she was completely asymptomatic. The echocardiogram at this visit revealed a thickened pericardium but no evidence of re-accumulation or constriction.

\section{Introduction and Background}

Although there has been some encouraging progress in the field of tuberculosis, such as the promising recent outcome of a phase $2 \mathrm{~b}$ placebo-controlled trial of the $\mathrm{M} 72 / \mathrm{AS} 01_{\mathrm{E}} \mathrm{TB}$ vaccine $[1 \bullet \cdot$, a great deal remains to be done. The statement is particularly true for tuberculous pericarditis (TBP), where lack of understanding of this severe form of extra-pulmonary tuberculosis (EPTB) has hampered discovery and translation of cost-effective, rapid diagnostic tests and host-directed therapies able to prevent its debilitating complications and associated mortality. The presence of HIV contributes considerably to the complexity of the disease process - both at the immunological and clinical levels.

\section{Epidemiology}

TB causes more deaths than any other infectious disease globally with the WHO estimating that there were 1.3 million TB deaths in HIV-uninfected individuals and 300,000 HIV-TB co-infected deaths in 2017 alone. This may be an underestimate of HIV co-infected TB deaths, as post-mortem studies of HIV-infected individuals in regions where TB is endemic have indicated that $45.8 \%$ of TB cases are undiagnosed at the time of death, and $87.9 \%$ of all deaths were due to disseminated TB [2].

The actual incidence of TBP is variable depending on the degree of TB endemicity of the region. In a case series from Spain, for example, only $4 \%$ of 294 admissions for the acute pericardial disease were due to TBP [3]. In Africa, pericardial effusions are predominantly due to $\mathrm{TB}$, ranging from 64.9 to $70 \%$ in various reports $[4,5]$. The HIV pandemic contributes to the high burden of TBP seen especially in Southern Africa, as illustrated by a study that included $84 \mathrm{HIV}$-infected individuals with effusive pericarditis - only 3 of these individuals had a diagnosis other than TB [5].

There is very little new data to inform the current epidemiology of TBP in children. However, considering that EPTB accounts for $21-44 \%$ of all TB in children, it is likely an under-recognized problem [6]. A recent prospective observational study from Papua New Guinea reported a case fatality rate of 25\% (4 out of 16) in children with TBP [7]. The most recently published retrospective observational study of children with TBP reported only one death out of 30 children, with the cause of death being ascribed to disseminated TB. The authors acknowledge that actual mortality might have been underestimated due to loss to follow-up [8]. The study reported that $10 \%$ of children developed constrictive pericarditis. This is in contrast to an earlier retrospective study of 44 pediatric TBP cases which reported no deaths and 5 cases (20\%) of constrictive pericarditis at follow-up [9]. There is a need for further research in pediatric TBP, especially considering the disproportionate impact of EPTB on this vulnerable population.

\section{Pathogenesis}

There has been some progress in our understanding of the pathogenesis of TBP, particularly as it relates to the disease in HIV-infected hosts. Mycobacterium tuberculosis (Mtb) bacilli can enter the pericardium by retrograde lymphatic spread, hematogenous dissemination, or, uncommonly, by direct contiguous spread from adjacent infected structures such as the lungs, pleura, and spine [10]. The most common mechanism of Mtb spread in HIV co-infected individuals is via hematogenous dissemination [11], whereas in HIV-uninfected TBP patients, pericardial fluid (PCF) lymphocytes are predominantly CD4+ effector memory $\mathrm{T}$ cells and the PCF lymphocytes in HIV-infected patients are dominated by CD8+ T cells $[12,13]$. HIV viral load is higher in PCF than in plasma of HIV-positive TBP patients and inversely correlates with the proportion of PCF CD4+ T cells [13]. The cellular influx into the PCF is accompanied by a range of predominantly proinflammatory and pro-fibrotic mediators (e.g., interferon- $\gamma$, interleukin (IL)-10, IL-1 $\beta$, IL-6, IL-8, interferon- $\gamma$ induced protein, and tumor necrosis factor (TNF)), that accumulate in the pericardial fluid $[14,15]$, and low levels of the antifibrotic tetra peptide $N$-acetylseryl-aspartyl-lysyl proline (AcSDKP) [16]. AcSDKP is broken down by angiotensinconverting enzyme [17], suggesting a novel role for ACE inhibitors for TBP to prevent pericardial fibrosis. Finally, recent reports suggest that among both HIV-infected and HIVuninfected patients with culture-positive pericardial fluid, Mtb bacillary loads are as high as $3.91 \log _{10} \mathrm{CFU} / \mathrm{mL}$ (range $0.5-$ 8.96 ), with bacillary loads over $5.53 \log _{10} \mathrm{CFU} / \mathrm{mL}$ being 
significantly associated with mortality [18]. These findings are significant as they challenge the commonly accepted concept that TBP is predominantly a paucibacillary condition driven by an intense delayed-type hypersensitivity response to TB antigens [10]. In at least some subsets of patients, TBP is, in fact, multibacillary with significant implications for both outcomes and ensuring that current drug regimens are effective at penetrating the pericardium to do their work.

There are four pathological phases of TBP that correspond with the various clinical manifestations observed (Table 1) [11].

Two broad mechanisms drive the clinical presentation of pericarditis: fluid accumulation within the pericardium which compresses the heart chambers throughout the cardiac cycle impeding both cardiac filling and cardiac contraction (tamponade); or thickening of the pericardium with minimum or absent effusion resulting in impairment of cardiac filling in diastole (constriction). The predominant clinical manifestation of TBP is the syndrome of heart failure regardless of mechanism although a relatively small proportion of patients with tamponade may also have hemodynamic compromise with hypotension tachycardia and shock.

New registries and trials suggest that the most common clinical presentation of TBP is as effusive pericarditis (79.5\%) [19] corresponding to stage 2. Constrictive pericarditis, which corresponds to stages 3 and 4 , is one of the most severe sequelae of TBP (Fig. 1). Before the widespread use of ATT and routine evacuation of the pericardium, constrictive pericarditis was found in 30-60\% of patients [3,20], whereas more modern prospective studies report rates of $5-25 \%[21 \bullet \bullet]$.
Table 1 Stages of tuberculous pericarditis

\author{
Stage 1 \\ - Pathological bases \\ - Pathological \\ manifestations \\ - Clinical manifestation \\ Stage 2 \\ - Pathological bases \\ - Pathological \\ manifestation \\ - Clinical manifestation
}

Stage 3

- Pathological bases

- Pathological

manifestation

-Clinical manifestation

Stage 4

- Pathological bases

- Pathological

manifestation

- Clinical manifestation
Fibrinous exudation predominates; occurrence of polymorphonuclear leucytosis is first seen with relatively abundant mycobacteria. There is a loose organization of macrophages and T cells with early granuloma formation (HIV patients with low CD4 T cells with fewer granuloma due to low immune response)

Dry stage (the least common form seen)

Patients present acute pericarditis with chest pain, pericardial friction rub, and widespread ST elevation without effusion

There are predominantly lymphocytic exudates with monocytes and foam cells; presence of serosanguineous effusion is seen

Effusive stage (most common form seen)

(1) Patients present with features of heart failure and/or cardiac tamponade due to moderate to large pericardial effusion

(2) Effusive constrictive pericarditis with coexistence of visceral constrictive pericarditis and simultaneous compressive pericardial fluid. The former become obvious following pericardial drainage

At this stage, there is absorption of effusion, granulomatous caseation becomes organized and perocardial thickening occurs due to fibrin deposition of collagen, and ultimately fibrosis

Adsorptive stage

Symptoms and signs compatible with constrictive perocarditis but radiological and echocardiographic evidence of thick fibrinous fluid around the heart

Constructive scarring (the fibrosing visceral and parietal pericardium contracts on the cardiac chambers). Calcification leads to encasing of the heart in a fibrocalcific skin. Diastolic filling is impeded, causing the classic syndrome of constrictive pericarditis

Constrictive stage

Constrictive pericarditis symptoms and signs predominate; and echocardiography confirms the diagnosis with no residual fluid in the pericardium

Table created based on information from ref. [11] 
Fig. 1 Cardiac magnetic resonance images in TB pericarditis. a T2-weighted STIR imaging with thickened visceral and parietal pericardium. b

Fibrotic pericardial layers after administration of gadolinium (reproduced with permission from Ntusi et al. [56])

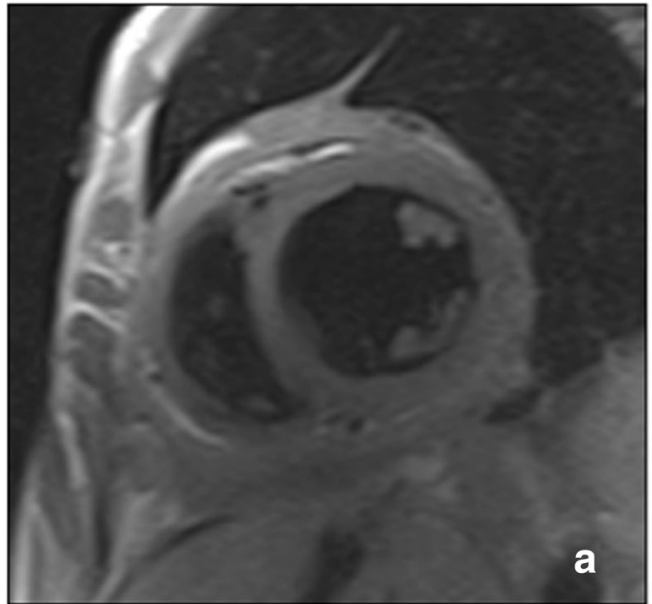

It occurs less frequently in those with HIV infection [22]. Some patients present with a combination of both tamponade and constrictive physiology believed to be a stage between 2 and 3 (effusive-constrictive) and highlighting that despite the discussion of these as separate clinical entities, they may overlap in clinical practice $[19,23]$.

\section{Diagnosing Tuberculous Pericarditis}

The most challenging aspect of the diagnosis of TBP remains to be establishing a tuberculous etiology. Despite best efforts, 15-20\% of the pericardial disease are never diagnosed [24], reflecting the overall paucity in reliable, cost-effective new diagnostic tests that can rapidly aid clinical decision-making. Adenosine deaminase (ADA) remains the most widely used biochemical test even though it is limited as a rule in test [25], and the yields from fluid microscopy and culture remain both low and time-consuming. The result is that the practice in many high TB burden regions has been to treat for TB empirically [19] despite observational evidence that empiric therapy is associated with higher morbidity and mortality [26]. For ease of diagnosis in TB endemic countries, some authors have developed criteria for diagnosis of definitive and probable TBP [27] based on clinical and laboratory findings (Table 2).

Pericardiocentesis, when feasible (effusion $>1 \mathrm{~cm}$ ), remains an essential part of the approach to patients with suspected TBP. It provides not only therapeutic but also diagnostic benefit [28]. A typical finding from the aspirated fluid is a protein-rich lymphocytic exudate that is frequently macroscopically bloodstained. Light's criteria remain the most helpful to distinguish between transudates (usually heart failure) and exudates [29].

Culture of pericardial fluid remains the most widely used diagnostic test for TBP with sensitivity ranging between 53 and $75 \%$; however, it takes at least 3 weeks to yield results [30]. There have been no new applicable technologies or methods to increase the reduced yield of pericardial fluid smear for acid-fast bacilli (AFB); however, the high specificity of positive microscopy may justify its continued use [31]. Concomitant HIV infection does seem to affect the yield of pericardial fluid culture. A recent study involving predominantly HIV-infected participants (72\%), and making use of liquid culture media only, detected culture positivity in 46 out of $131(35 \%)$ of pericardial fluid specimens [31]. Where there is diagnostic doubt despite biochemical ADA and fluid culture, or where the pericardial fluid is difficult to obtain or in the research setting, a biopsy of the pericardium or epicardium may be warranted $[32 \cdot, 33]$. However, there are no new techniques or tools to increase the yield from histological analysis, which remains $10-64 \%$ [27]. What is new is that problems with obtaining representative pericardial biopsy samples could potentially be overcome by using percutaneous pericardioscopy. A small pilot study using the technique demonstrated a diagnostic yield of $60 \%$ using AFB staining of
Table 2 Diagnostic criteria for TB pericarditis in TB endemic countries

\begin{tabular}{ll}
\hline Definite TB pericarditis & Probable TB pericarditis \\
\hline $\begin{array}{c}\text { Tubercle bacilli are found in stained smear or culture of } \\
\text { pericardial fluid; and/or } \\
\begin{array}{c}\text { Tubercle bacilli or caseating granulomata are found on } \\
\text { histological examination of the pericardium }\end{array}\end{array}$ & $\begin{array}{c}\text { Evidence of pericarditis in a patient with tuberculosis } \\
\text { demonstrated elsewhere in the body; and/or }\end{array}$ \\
& $\begin{array}{c}\text { Lymphocytic pericardial exudate with elevated ADA } \\
\text { activity; and/or } \\
\text { Good response to anti-tuberculosis chemotherapy }\end{array}$ \\
\hline
\end{tabular}

Table created based on information from ref. [27] 
biopsy samples, with an additional $13.3 \%$ being diagnosed by identification of granulomatous inflammation on histology [30].

Xpert MTB/RIF and Xpert MTB/RIF Ultra are cartridgebased nucleic acid amplification tests that can detect Mtb and simultaneously test for rifampicin resistance in under $2 \mathrm{~h}$. In a study assessing the accuracy of Xpert MTB/RIF in TBP and other forms of extrapulmonary TB, the sensitivity was $63.8 \%$, with high specificity (100\%) [34]. Elsewhere, use of Xpert MTB/RIF on pericardial biopsy samples increased the sensitivity to $78 \%$ [35••]. These results do not show much improvement from much older studies on polymerase chain reaction (PCR)-based techniques [36]. Finally, it is notable that the use of Xpert MTB/RIF in combination with ADA does increase the sensitivity and overall accuracy of the diagnosis of TBP and could be considered a diagnostic option where there is major diagnostic uncertainty [37]. The associated costs of such a strategy are high, perhaps explaining the lack of widespread uptake of such a strategy among clinicians.

Gamma-interferon (IFN- $\gamma$ ) is produced in large amounts by both CD-4+ and CD-8+ T lymphocytes in the setting of TBP, which has been demonstrated to be highly accurate as a diagnostic biomarker since it was first evaluated [38-40]. A recent meta-analysis of four published studies found an overall sensitivity (97\%) and specificity (99\%) of PCF IFN- $\gamma$ to diagnose TBP, in addition to a positive likelihood ratio of 187 and negative likelihood ratio of 0.03 , which provides strong evidence that PCF IFN- $\gamma$ could be used as a stand-alone test to diagnose or exclude TBP [41••]. Despite its clear superiority, IFN- $\gamma$ is not a widely available commercial test in diagnostic laboratories in endemic areas due to high cost.

Tuberculin skin test (TST) and interferon-gamma release assays (IGRAs) are of limited value in areas of high TB endemicity as positivity of either merely reflects exposure to Mtb antigens and cannot accurately discern active TB disease [42]. Furthermore, the purified protein derivative (PPD) used by the TST can yield false-positive results in individuals vaccinated with bacilli Calmette-Guerin (BCG) and in those who have been sensitized to nontuberculous mycobacteria (NTM).

\section{Management of Tuberculous Pericarditis}

The treatment of TBP is aimed at achieving three goals: killing and control of active Mtb; relief of the cardiac compression and adverse hemodynamic sequelae (tamponade and heart failure); and the prevention of complications of maladaptive pericardial remodeling and healing, including constrictive pericarditis.

The goal of effective killing and control of Mtb using four anti-tuberculous drug regimens (rifampicin, isoniazid, ethambutol, and pyrazinamide) for a minimum of 6 months has been the standard of care for decades in the absence of evidence of comparative effectiveness. This is important because of recent findings from a small pharmacokinetic and pharmacodynamic study, which demonstrated that there is low penetration of anti-TB drugs in the pericardial space over the first $24 \mathrm{~h}$ following drug administration. The median peak pericardial fluid rifampicin concentrations were significantly lower than the minimum inhibitory concentration (MIC) [43]. In the same study, pyrazinamide peak concentration was 40 times lower than the $\mathrm{pH}$ adjusted MIC. Isoniazid was the only drug found at adequate concentrations in the pericardial fluid of patients with TBP. These findings suggest that there is an urgent need to investigate higher doses or alternative drug regimens in patients with TBP, in whom poor drug penetration may contribute to the poor outcomes observed. This is particularly true considering recent evidence of the close relationship between the burden of bacillary load within the pericardium and mortality [18].

Echocardiographic- or fluoroscopic-guided needle pericardiocentesis remains the treatment of choice to evacuate the pericardium of compressive pericardial fluid and alleviate cardiac tamponade. The European Society of Cardiology (ESC) Working Group on Myocardial and Pericardial Diseases have developed a triage system based on the opinion of experts which can be used to guide clinical decisionmaking in the management of patients with cardiac tamponade [44]. There are still no studies comparing routine evacuation of the pericardium and evacuation only for clinical tamponade. However, the recent characterization of the pericardial fluid with toxic proinflammatory and profibrotic mediators has added to the list of theoretical benefits the manual removal of these mediators to prevent short- and long-term damage to the pericardium. There is also good circumstantial evidence that in recent prospective studies with high rates of pericardiocentesis $[21,45,46]$, the rates of constrictive pericarditis are lower (5-15\%) than the rates (17-30\%) quoted from eras when there was less aggressive use of the intervention $[3,20]$.

The goal of preventing pericardial fibrosis and constrictive pericarditis is of importance in sub-Saharan African. This is because the syndrome is an important and relatively common cause of heart failure in the region [47], where the facilities, expertise, and experience needed to perform definitive surgical pericardiectomy are virtually non-existent [48]. Interventions for the prevention of constrictive pericarditis which have been tested or are being tested include corticosteroids, Mycobacterium indicus pranii, colchicine, and fibrinolytic therapy.

Four randomized controlled trials $[21 \bullet \bullet, 46,49,50]$ have evaluated the role of intrapericardial and oral corticosteroids for the prevention of progression to constriction. In a recent Cochrane review of the 4 randomized trials [51], analysis of the cumulative data suggested that there was low certainty of evidence for a $20 \%$ reduction in all-cause mortality (risk ratio (RR) 0.80 , 95\% confidence interval (CI) $0.59-1.09$; 660 
participants), compared with those who did not receive steroids in whom a significant reduction in pericarditis-related deaths was reported (RR 0.39, 95\% CI 0.19-0.80; 660 participants). However, among HIV-positive participants, in the large and most robust of the studies, no evidence for a reduction in mortality was found with a possible increase in the rates of HIV/AIDS-related malignancy in those with advanced HIV but who were not yet on ART at enrolment [52・•]. The same study showed a reduction in the risk of constrictive pericarditis and rehospitalizations in participants on corticosteroids independent of HIV status [21••]. Based on these findings, the European Society of Cardiology pericardial disease guidelines recommend that it may be reasonable to use adjunctive corticosteroids at a tapering dose of $120 \mathrm{mg}$ of prednisolone over 6 weeks in patients with TBP without HIV infection, and to avoid their use in HIV-infected individuals because of the increased risk of malignancy [28]. The use of $M$. indicus pranii immunotherapy was evaluated in the same RCT by factorial design and was found to be neutral on all efficacy outcomes in HIV-negative participants, with increased malignancy in the subset with advanced HIV but not on ART $[21 \bullet \cdot]$.

In an underpowered South African study, investigators tested whether the positive impact of the drug colchicine on preventing complications in patients with idiopathic pericarditis [53] could be extended to patients with TBP. They tested the hypothesis that colchicine taken daily for 6 weeks may reduce the rates of constrictive pericarditis relative to a placebo control arm receiving "usual care." The results were neutral, with no benefit or harm found [54].

Finally, intrapericardial fibrinolysis is currently under investigation in the Second Investigation of the Management of Pericarditis (IMPI-2) Trial (https://clinicaltrials.gov/ct2/show/ NCT02673879). The rationale for its use is based on its theoretical ability to break up loculated fibrin strands allowing for complete evacuation of the pericardium and reduction in mediators of fibrosis; and 60 years of experience from case reports and small case series where success rates have been high [55••]. The multicenter trial is currently in its feasibility phase.

\section{Conclusion}

Tuberculous pericarditis remains a major cause of cardiovascular death and disability in TB endemic regions of the world, particularly where the prevalence of HIV is high. Historically, contributors to this high morbidity and mortality have included the following: little progress in our understanding of the immunopathogenesis of the disease in order to identify potential targets for therapy; failure to develop a relatively inexpensive, rapid, accurate, and widely available test that can be used out in the field at the point of contact in patients with the suspected disease; a better understanding of the determinants and drivers of post-tuberculous fibrosis and constrictive pericarditis; more detailed understanding of the pharmacokinetic and pharmacodynamics of anti-tuberculous drug in both plasma and the pericardium and its impact on short- and long-term outcomes. In this review, we have highlighted areas where there have been significant developments, such as a more detailed study of the immune pathways, cells, and cytokine mediators of the response to TB; those such as diagnosis of tuberculous etiology, and interventions to reduce mortality and morbidity where progress has been elusive; and described those areas where there may be opportunities to turn the tide on this burdensome disease.

\section{Compliance with Ethical Standards}

Conflict of Interest Godsent Isiguzo, Elsa Du Bruyn, Patrick Howlett, and Mpiko Ntsekhe declare that they have no conflict of interest.

Human and Animal Rights and Informed Consent This article does not contain any studies with human or animal subjects performed by any of the authors.

\section{References}

Papers of particular interest, published recently, have been highlighted as:

- Of importance

•- Of major importance

1.• Van Der Meeren O, et al. Phase $2 \mathrm{~b}$ controlled trial of M72/AS01E vaccine to prevent tuberculosis. N Engl J Med. 2018;379(17): 1621-34. The article provides hope for eradication of tuberculosis by the finding that M72/AS01E provided 54.0\% protection for M. tuberculosis-infected adults against active pulmonary tuberculosis disease, without evident safety concerns.

2. Gupta RK, Lucas SB, Fielding KL, Lawn SD. Prevalence of tuberculosis in post-mortem studies of HIV-infected adults and children in resource-limited settings: a systematic review and meta-analysis. Aids. 2015;29(15):1987-2002.

3. Sagrista-Sauleda J, Permanyer-Miralda G, Soler-Soler J. Tuberculous pericarditis: ten year experience with a prospective protocol for diagnosis and treatment. J Am Coll Cardiol. 1988;11(4):724-8.

4. Mynors JM, Reichman B. Pericarditis-a five year study in the African. Cent Afr J Med. 1973;19(2):19-22.

5. Reuter H, Burgess LJ, Doubell AF. Epidemiology of pericardial effusions at a large academic hospital in South Africa. Epidemiol Infect. 2005;133(3):393-9.

6. Nelson LJ, Wells CD. Global epidemiology of childhood tuberculosis. Int J Tuberc Lung Dis. 2004;8(5):636-47.

7. Watch $\mathrm{V}$, et al. The burden of presumed tuberculosis in hospitalized children in a resource-limited setting in Papua New Guinea: a prospective observational study. Int Health. 2017;9(6):374-8.

8. Obihara NJ, et al. Tuberculous pericardial effusions in children. J Pediatric Infect Dis Soc. 2018;7(4):346-9. 
9. Hugo-Hamman CT, Scher H, De Moor MM. Tuberculous pericarditis in children: a review of 44 cases. Pediatr Infect Dis J. 1994;13(1):13-8.

10. Spodick DH. Tuberculous pericarditis. AMA Arch Intern Med. 1956;98(6):737-49.

11. Ntsekhe M, Mayosi BM. Tuberculous pericarditis with and without HIV. Heart Fail Rev. 2013;18(3):367-73.

12. Reuter $\mathrm{H}$, et al. Characterization of the immunological features of tuberculous pericardial effusions in HIV positive and HIV negative patients in contrast with non-tuberculous effusions. Tuberculosis (Edinb). 2006;86(2):125-33.

13. Matthews K, Ntsekhe M, Syed F, Scriba T, Russell J, Tibazarwa K, et al. HIV-1 infection alters CD4+ memory T-cell phenotype at the site of disease in extrapulmonary tuberculosis. Eur $\mathrm{J}$ Immunol. 2012;42(1):147-57.

14. Shenje J, et al. Effect of prednisolone on inflammatory markers in pericardial tuberculosis: a pilot study. Int J Cardiol Heart Vasc. 2018;18:104-8.

15. Matthews K, Deffur A, Ntsekhe M, Syed F, Russell JB, Tibazarwa $\mathrm{K}$, et al. A compartmentalized profibrotic immune response characterizes pericardial tuberculosis, irrespective of HIV-1 infection. Am J Respir Crit Care Med. 2015;192(12):1518-21.

16. Ntsekhe M, Matthews K, Wolske J, Badri M, Wilkinson KA, Wilkinson RJ, et al. Ac-SDKP (N-acetyl-seryl-aspartyl-lysyl-proline) and galectin-3 levels in tuberculous pericardial effusion: implications for pathogenesis and prevention of pericardial constriction. Heart. 2012;98(17):1326-8.

17. Mnguni AT, Engel ME, Borkum MS, Mayosi BM. The effects of angiotensin converting enzyme inhibitors (ACE-I) on human Nacetyl-seryl-aspartyl-lysyl-proline (Ac-SDKP) levels: a systematic review and meta-analysis. PLoS One. 2015;10(12):e0143338.

18. Pasipanodya JG, et al. Tuberculous pericarditis is multibacillary and bacterial burden drives high mortality. EBioMedicine. 2015;2(11): 1634-9.

19. Mayosi BM, et al. Clinical characteristics and initial management of patients with tuberculous pericarditis in the HIV era: the Investigation of the Management of Pericarditis in Africa (IMPI Africa) registry. BMC Infect Dis. 2006;6:2.

20. Schrire V. Experience with pericarditis at Groote Schuur Hospital, Cape Town: an analysis of one hundred and sixty cases studied over a six-year period. S Afr Med J. 1959;33:810-7.

21.• Mayosi BM, et al. Prednisolone and Mycobacterium indicus pranii in tuberculous pericarditis. N Engl J Med. 2014;371(12):1121-30

This landmark RCT was the first in Africa to investigate the place of steriod in tuberculous pericarditis. The results show that among HIV negative patients with TBP, prednisolone led to reduced incidence of constrictive pericarditis.

22. Ntsekhe M, Wiysonge CS, Gumedze F, Maartens G, Commerford PJ, Volmink JA, et al. HIV infection is associated with a lower incidence of constriction in presumed tuberculous pericarditis: a prospective observational study. PLoS One. 2008;3(6):e2253.

23. Syed FF, Mayosi BM. A modern approach to tuberculous pericarditis. Prog Cardiovasc Dis. 2007;50(3):218-36.

24. Noubiap JJ, et al. Epidemiology of pericardial diseases in Africa: a systematic scoping review. Heart. 2019;105(3):180-8.

25. Xie D, Cheng B, Sheng Y, Jin J. Diagnostic accuracy of adenosine deaminase for tuberculous pericarditis: a meta-analysis. Eur Rev Med Pharmacol Sci. 2015;19(22):4411-8.

26. Mayosi BM, et al. Mortality in patients treated for tuberculous pericarditis in sub-Saharan Africa. S Afr Med J. 2008;98(1):36-40.

27. Mayosi BM, Burgess LJ, Doubell AF. Tuberculous pericarditis. Circulation. 2005;112(23):3608-16.

28. Adler Y, Charron P, Imazio M, Badano L, Barón-Esquivias G, Bogaert J, et al. 2015 ESC guidelines for the diagnosis and management of pericardial diseases: The Task Force for the Diagnosis and Management of Pericardial Diseases of the European Society of Cardiology (ESC) endorsed by: the European Association for Cardio-Thoracic Surgery (EACTS). Eur Heart J. 2015;36(42): 2921-64.

29. Light RW. Pleural effusions. Med Clin North Am. 2011;95(6): 1055-70.

30. Kyriakakis CG, Mayosi BM, de Vries E, Isaacs A, Doubell AF. An approach to the patient with suspected pericardial disease. SAMJ: South African Medical Journal. 2016;106(2):151-5.

31. Theron G, et al. Determinants of PCR performance (Xpert MTB/ RIF), including bacterial load and inhibition, for TB diagnosis using specimens from different body compartments. Sci Rep. 2014;4: 5658.

32. Kyriakakis CG. Tuberculous pericarditis: challenges and controversies in the modern era. SA Heart. 2016;13(2):104-11. One of the issues that delay early commencement of treatment in TBP is definitive diagnosis. The authors of the article introduce use of a pericardioscope for pericardial tissue collection to aid tissue diagnosis.

33. Corey GR, Campbell PT, van Trigt P, Kenney RT, O'Connor CM, Sheikh KH, et al. Etiology of large pericardial effusions. Am J Med. 1993;95(2):209-13.

34. Pandie S, et al. Diagnostic accuracy of quantitative PCR (Xpert MTB/RIF) for tuberculous pericarditis compared to adenosine deaminase and unstimulated interferon- $\gamma$ in a high burden setting: a prospective study. BMC Med. 2014;12(1):101.

35. Yu G, et al. Comparison between the diagnostic validities of Xpert $\mathrm{MTB} / \mathrm{RIF}$ and interferon-gamma release assays for tuberculous pericarditis using pericardial tissue. PLoS One. 2017;12(12): e0188704. The World Health Organization has identified development of point of care diagnostic tools as the key in improvement of diagnosis and outcome in tuberculosis. The authors of this article show the high sensitivity of Xpert MTB/RIF in TBP. However, its non-superiority compared with older PCR is a drawback and calls for further investigations.

36. Cegielski JP, Devlin BH, Morris AJ, Kitinya JN, Pulipaka UP, Lema LE, et al. Comparison of PCR, culture, and histopathology for diagnosis of tuberculous pericarditis. J Clin Microbiol. 1997;35(12):3254-7.

37. Pandie S, et al. Diagnostic accuracy of quantitative PCR (Xpert MTB/RIF) for tuberculous pericarditis compared to adenosine deaminase and unstimulated interferon-gamma in a high burden setting: a prospective study. BMC Med. 2014;12:101.

38. Bian S, Zhang Y, Zhang L, Shi X and Liu X. Diagnostic Value of Interferon-gamma Release Assays on Pericardial Effusion for Diagnosis of Tuberculous Pericarditis. PLoS One. 2016;11: e0165008

39. Burgess LJ, et al. The use of adenosine deaminase and interferongamma as diagnostic tools for tuberculous pericarditis. Chest. 2002;122(3):900-5.

40. Latouf S, Levetan B, Commerford P. Tuberculous pericardial effusion: analysis of commonly used diagnostic methods. S Afr Med J. 1996;86(Suppl):15.

41. Liu C, et al. Diagnostic accuracy of interferon-gamma in pericardial effusions for tuberculous pericarditis: a meta-analysis. J Thorac Dis. 2018;10(2):854-60. The authors highlight the role of interferon gamma as a stand-alone test to exclude or diagnose TBP, which is a potential step in rapid diagnosis, with the high cost a drawback to its use in low-medium-income countries (LMICs).

42. Cattamanchi A, Smith R, Steingart KR, Metcalfe JZ, Date A, Coleman $\mathrm{C}$, et al. Interferon-gamma release assays for the diagnosis of latent tuberculosis infection in HIV-infected individuals: a systematic review and meta-analysis. J Acquir Immune Defic Syndr. 2011;56(3):230-8.

43. Shenje $\mathrm{J}$, et al. Poor penetration of antibiotics into pericardium in pericardial tuberculosis. EBioMedicine. 2015;2(11):1640-9. 
44. Ristic AD, et al. Triage strategy for urgent management of cardiac tamponade: a position statement of the European Society of Cardiology Working Group on Myocardial and Pericardial Diseases. Eur Heart J. 2014;35(34):2279-84.

45. Liebenberg J, van der Bijl P. A "vanishing", tuberculous, pericardial effusion. Korean Circ J. 2016;46(6):879-81.

46. Reuter H, Burgess LJ, Louw VJ, Doubell AF. The management of tuberculous pericardial effusion: experience in 233 consecutive patients. Cardiovasc J S Afr. 2007;18(1):20-5.

47. Damasceno A, et al. The causes, treatment, and outcome of acute heart failure in 1006 Africans from 9 countries. Arch Intern Med. 2012;172(18):1386-94.

48. Yankah C, et al. Cardiac surgery capacity in sub-Saharan Africa: quo vadis? Thorac Cardiovasc Surg. 2014;62(5):393-401.

49. Strang JI, Kakaza HH, Gibson DG, Girling DJ, Nunn AJ, Fox W. Controlled trial of prednisolone as adjuvant in treatment of tuberculous constrictive pericarditis in Transkei. Lancet. 1987;2(8573): $1418-22$.

50. Strang JI, Nunn AJ, Johnson DA, Casbard A, Gibson DG, Girling DJ. Management of tuberculous constrictive pericarditis and tuberculous pericardial effusion in Transkei: results at 10 years followup. Qjm. 2004;97(8):525-35.

51. Wiysonge CS, et al. Interventions for treating tuberculous pericarditis. Cochrane Database Syst Rev. 2017;9:Cd000526.

52.• Mayosi BM, et al. Rationale and design of the Investigation of the Management of Pericarditis (IMPI) trial: a $2 \times 2$ factorial randomized double-blind multicenter trial of adjunctive prednisolone and Mycobacterium w immunotherapy in tuberculous pericarditis. Am
Heart J. 2013;165(2):109-15.e3. The authors of this Cochrane review provide a bird's-eye view of interventions in the past 5 years focused on treatment of tuberculous pericarditis, and reveal areas of gaps in knowledge as well as potential research areas.

53. Imazio M, Brucato A, Cemin R, Ferrua S, Belli R, Maestroni S, et al. Colchicine for recurrent pericarditis (CORP): a randomized trial. Ann Intern Med. 2011;155(7):409-14.

54. Liebenberg JJ, Dold CJ, Olivier LR. A prospective investigation into the effect of colchicine on tuberculous pericarditis. Cardiovasc J Afr. 2016;27(6):350-5.

$55 . \bullet$ Wiyeh $\mathrm{AB}$, et al. A systematic review of the efficacy and safety of intrapericardial fibrinolysis in patients with pericardial effusion. Int J Cardiol. 2018;250:223-8. The authors of this article posit that fibrin deposition could be central in the development of constrictive pericarditis and a potential target in its prevention. The ongoing Second Investigation of Management of Pericarditis (IMPI-2) Trial is designed to address this.

56. Ntusi NA, Palkowski G, Samuels P, Moosa S, Ntsekhe M, Mayosi B. Cardiovascular magnetic resonance characterisation of pericardial and myocardial involvement in patients with tuberculous pericardial constriction with and without HIV co-infection. J Cardiovasc Magn Reson. 2016;18:Q29. https://doi.org/10.1186/ 1532-429X-18-S1-Q29.

Publisher's Note Springer Nature remains neutral with regard to jurisdictional claims in published maps and institutional affiliations. 